\title{
First-Principles Study on Various Point Defects Formed by Hydrogen and Helium Atoms in Tungsten
}

\author{
Qiang Zhao, ${ }^{1} \mathrm{Zheng}^{\mathrm{Zhang}}{ }^{1}$ Yang Li, ${ }^{1}$ and Xiaoping Ouyang ${ }^{1,2,3}$ \\ ${ }^{1}$ Beijing Key Laboratory of Passive Safety Technology for Nuclear Energy, School of Nuclear Science and Engineering, \\ North China Electric Power University, Beijing 102206, China \\ ${ }^{2}$ Northwest Institute of Nuclear Technology, Xian 710024, China \\ ${ }^{3}$ School of Materials Science and Engineering, Xiangtan University, Xiangtan 411105, China
}

Correspondence should be addressed to Qiang Zhao; qzhao@ncepu.edu.cn

Received 2 April 2017; Revised 10 July 2017; Accepted 13 July 2017; Published 7 September 2017

Academic Editor: Eugenijus Ušpuras

Copyright (C) 2017 Qiang Zhao et al. This is an open access article distributed under the Creative Commons Attribution License, which permits unrestricted use, distribution, and reproduction in any medium, provided the original work is properly cited.

The different point defects formed by two hydrogen atoms or two helium atoms in tungsten were investigated through firstprinciples calculation. The energetically favorable site for a hydrogen atom is tetrahedral interstitial site while substitutional site is the most preferred site for a helium atom. The formation energies of two hydrogen or helium atoms are determined by their positions, and they are not simply 2 times the formation energy of a single hydrogen or helium atom's defect. After relaxation, two adjacent hydrogen atoms are away from each other while helium atoms are close to each other. The reasons for the interaction between two hydrogen or helium atoms are also discussed.

\section{Introduction}

Tungsten (W) is widely considered as the most potential plasma facing material (PFM) [1] and the first wall material (FWM) in fusion reactor due to its excellent properties, such as high melting point, high mechanical strength at high temperatures, good thermal conductivity, low thermal expansion coefficient, and high sputtering threshold energy [2]. Studies on tungsten receive an increasing amount of attention from scientists and engineers. Tungsten is subjected to high particle fluxes in fusion reactors, not only the plasma background ions $(\mathrm{H}, \mathrm{D}$, and $\mathrm{T})$ but also the intrinsic impurities $(\mathrm{He}, \mathrm{Be}$, and $\mathrm{C})$. Because hydrogen $(\mathrm{H})$ is one of the major plasma background ions, hydrogen irradiation may result in the change of the mechanical properties of tungsten. Helium $(\mathrm{He})$ irradiation leads to the blister formation and the subsequent degradation of the mechanical properties of metals. Thus, it is very important to investigate the trapping and blister formation of hydrogen or helium in tungsten and the behaviors of hydrogen and helium in tungsten.

A number of experimental and theoretical studies were carried out to understand the interaction between the hydrogen or helium impurity and the tungsten host lattice [1]. For example, Arkhipov et al. found that hydrogen could form a blister on the tungsten surface [3]. You presented the experimental results in a divertor plasma simulator NAGDIS-II and observed the formation of hydrogen bubbles on the surface of tungsten [4]. Grigorev et al. observed the nucleation and growth of hydrogen bubbles on dislocations in tungsten under high-flux, low energy plasma exposure [5]. Liu et al. studied the behavior of hydrogen in tungsten through firstprinciples calculation [6]. Wang et al. studied the behavior of helium on tungsten surfaces and the hydrogen in tungsten by using first-principles calculation [7]. Li et al. studied the behavior of hydrogen or helium in tungsten by using the molecular dynamics simulation method [8]. With the rapid development of the computer technique, the first-principles calculation method plays an important role in the materials research; it can investigate the mechanical properties, electronic structure, and optical properties of some new kinds of materials [9-11].

There are a lot of works focused on defects in tungsten. For example, research work on the interaction between two hydrogen atoms that are located in tetrahedral interstitial 


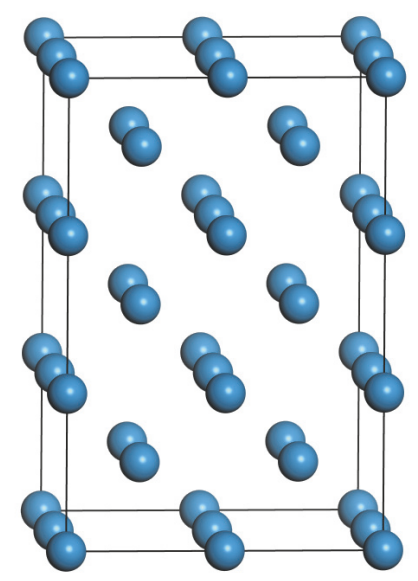

(a)

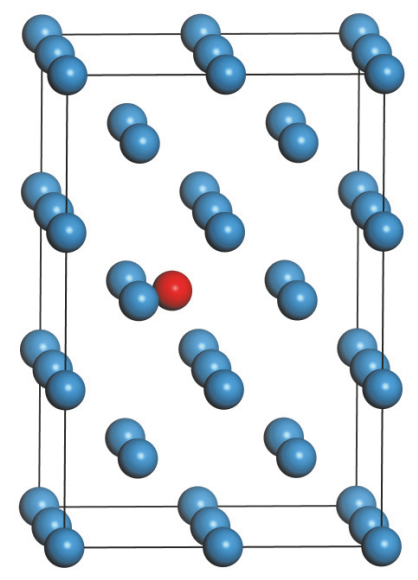

(c)

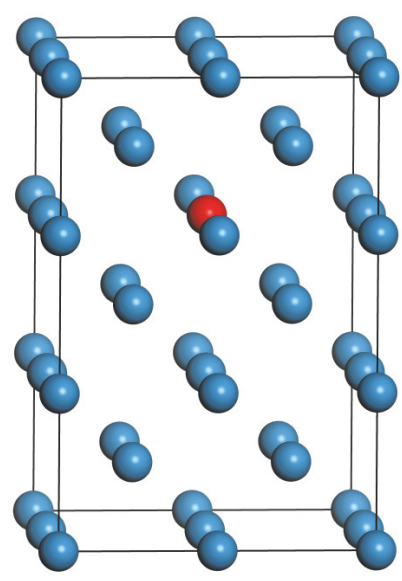

(b)

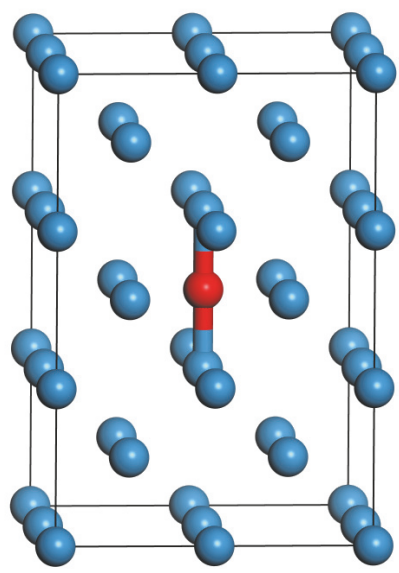

(d)

FIgURE 1: The crystal structure of BCC W. The blue balls stand for the tungsten atoms, and the red ball stands for the H atom or He atom. A pure W supercell (a) and a W supercell contain a $\mathrm{H}$ atom or He atom in the substitutional site (b), tetrahedral site (c), and octahedral site (d).

sites in tungsten has been completed [12]. Some researchers investigated the interaction between hydrogen or helium atoms and the vacancy [1]. However, a systematic study of the interaction between two hydrogen or helium atoms in tungsten without other defects is still lacked. In this paper, by using first-principles calculation method based on density functional theory (DFT), we have studied the behavior of hydrogen atoms and helium atoms in body centered cubic (BCC) tungsten. We calculated the formation energy of single hydrogen atom and two hydrogen atoms in tungsten and the formation energy of single helium atom and two helium atoms in tungsten. Using the charge density difference and atomic population, we analyzed the reason why there is a repulsive interaction between two hydrogen atoms and an attractive interaction between two helium atoms. Our computational results might shed some light on the development of tungsten-based materials that are used in ITER and other fusion rectors. For example, Cheng's research group investigated the blistering behavior and deuterium retention in tungsten exposed to high-flux deuterium-neon mixed plasmas by experiment research [13] (the temperature of target surface is $10 \mathrm{~K}$ ) after a lot of first-principles calculations were completed; the results of Nguyen-Manh and Dudarev's [14] first-principles calculation are in good agreement with experimental data derived from thermal desorption spectroscopy [15] (annealing temperature is about $2150 \mathrm{~K})$.

\section{Models and Computational Details}

Tungsten belongs to the BCC crystal system, its space group is $\mathrm{Im}-3 \mathrm{~m}$. The simulation supercell composed of 24 lattice points $(2 \times 3 \times 2)$ was used in this paper. Initially, we considered three possible sites for a single $\mathrm{H}$ atom and $\mathrm{He}$ atom entering the lattice of BCC W. According to their positions, we marked the $\mathrm{H}$ and $\mathrm{He}$ atoms as $\mathrm{H}_{\text {Sub }}, \mathrm{H}_{\text {Tet }}, \mathrm{H}_{\mathrm{Oct}}, \mathrm{He}_{\mathrm{Sub}}, \mathrm{He}_{\text {Tet }}$, and $\mathrm{He}_{\mathrm{Oct}}$ when the $\mathrm{H}$ atom and $\mathrm{He}$ atom were located in the substitutional site, the tetrahedral interstitial site, and the octahedral interstitial site, respectively. The calculation models that contain a single $\mathrm{H}$ atom or a $\mathrm{He}$ atom are shown in Figure 1. To explore the interaction between two $\mathrm{H}$ atoms or two $\mathrm{He}$ atoms, we build a series of two $\mathrm{H}$ atoms or two $\mathrm{He}$ 
atoms models. The symbols $\mathrm{H}_{\mathrm{Sub}}^{21}, \mathrm{H}_{\mathrm{Sub}}^{22}$, and $\mathrm{H}_{\mathrm{Sub}}^{23}$ stand for the first-nearest-neighbor, second-nearest-neighbor, and thirdnearest-neighbor $\mathrm{H}$ atom around the preexisting $\mathrm{H}$ atom that is located in the substitutional site, and we use the symbols $\mathrm{H}_{\text {Tet }}^{21}, \mathrm{H}_{\text {Tet }}^{22}, \mathrm{H}_{\text {Tet }}^{23}, \mathrm{H}_{\text {Oct }}^{21}, \mathrm{H}_{\mathrm{Oct}}^{22}$, and $\mathrm{H}_{\text {Oct }}^{23}$ standing for two $\mathrm{H}$ atoms that are located in the different tetrahedral interstitial sites and octahedral interstitial sites, respectively. We use the symbols $\mathrm{He}_{\text {Sub }}^{21}, \mathrm{He}_{\text {Sub }}^{22}, \mathrm{He}_{\text {Sub }}^{23}, \mathrm{He}_{\mathrm{Tet}}^{21}, \mathrm{He}_{\mathrm{Tet}}^{22}, \mathrm{He}_{\mathrm{Tet}}^{23}, \mathrm{He}_{\text {Oct }}^{21}, \mathrm{He}_{\mathrm{Oct}}^{22}$, and $\mathrm{He}_{\mathrm{Oct}}^{23}$ standing for the first-nearest-neighbor, secondnearest-neighbor, and third-nearest-neighbor He around the preexisting He atom that is located in substitutional, tetrahedral, and octahedral sites, respectively. These models contain two $\mathrm{H}$ atoms or two $\mathrm{He}$ atoms and are shown in Figure 2.

First-principles calculations were performed by using the DFT [16] and the plane wave pseudo-potential technique, as implemented in the Cambridge Sequential Total Energy Package (CASTEP) [17]. The generalized gradient approximation (GGA) [18] with Perdew-Burke-Ernzerhof (PBE) [19] functional was used to describe the exchange-correlation interaction among the electrons, and ultrasoft pseudopotentials were employed for ion-electron interaction. For $\mathrm{W}$, the four $5 d$ electrons are considered to be the valence electrons together with two $6 s$ electrons (the reference state is $\left.5 d^{4} 6 s^{2}\right)$. During the calculation, the supercell size, shape, and atomic position are relaxed to equilibrium. The plane wave energy cutoff is $400 \mathrm{eV}$ for all calculations. The basic parameters were chosen as follows: the space representation is reciprocal, SCF tolerance equals $1.0 \times 10^{-6} \mathrm{eV} /$ atom, and $k$ sampling with $5 \times 5 \times 5 k$-point mesh in the Brillouin zone was used, after the convergence test was completed. The total energy of the supercell deceases as the plane wave cutoff and $k$-point mesh increasing; the structure also becomes more stable. Considering the optimization of computing resource, we set the plane wave cutoff and $k$-point mesh as mentioned above. Our parameters setting is in agreement with that of Liu's group [20]; our calculated formation energy of a $\mathrm{H}$ or $\mathrm{He}$ atom in $\mathrm{W}$ is in good agreement with Liu et al.s results. Moreover, the increase of these two calculation parameters does not significantly change the total energy of the supercell. The optimal atomic positions were determined by satisfying the following conditions: (1) the maximum interatomic forces were smaller than $0.05 \mathrm{eV} / \mathrm{nm}$; (2) the maximum change of energy per atom was smaller than $1.0 \times 10^{-5} \mathrm{eV}$; (3) the maximum displacement was smaller than $0.001 \AA$; and (4) the maximum stress of the crystal was smaller than $0.02 \mathrm{GPa}$. All the properties that we calculated based on the crystal structure have been optimized.

\section{Results and Discussion}

3.1. Optimization of the Crystal Structure. In order to study various defects formed by $\mathrm{H}$ or $\mathrm{He}$ atoms in $\mathrm{W}$, first of all, we optimized the crystal structure of the W supercell with and without the various defects formed by $\mathrm{H}$ or $\mathrm{He}$ atoms. Table 1 shows the calculated results, lattice parameters, elastic constants, and elastic modulus of the BCC W. The result shows that our results are in good agreement with the theoretical simulation results [21] and the experimental data [22]. Figure 3 shows the change of the volume of BCC W when the $\mathrm{H}$ or He atoms were introduced in W. From Figure 3, we can find that the volume of $\mathrm{W}$ decreases when the $\mathrm{H}$ or He atoms are located in the substitutional sites while the volume increases when the $\mathrm{H}$ or $\mathrm{He}$ atoms are located in the tetrahedral and octahedral sites. The reason for the volume decrease is that the atomic radius of $\mathrm{H}$ or $\mathrm{He}$ is much smaller than that of $\mathrm{W}$, and the volume increases due to the extra atoms that enter the $\mathrm{W}$ supercell.

3.2. Formation Energy. The formation energy of various defect configurations, $E_{\text {defect }}^{\mathrm{f}}$, was defined as follows:

$$
E_{\text {defect }}^{\mathrm{f}}=E_{\text {system }}^{\mathrm{tot}}-E_{\mathrm{W}}^{24}\left(\frac{N}{24}\right)-n_{\mathrm{He}} E_{\mathrm{He}}-n_{\mathrm{H}} E_{\mathrm{H}}
$$

where $E_{\text {system }}^{\text {tot }}$ is the total energy of the system with defects, $N, n_{\mathrm{He}}$, and $n_{\mathrm{H}}$ are the numbers of $\mathrm{W}, \mathrm{He}$, and $\mathrm{H}$ atoms, respectively, $E_{\mathrm{W}}^{24}$ is the total energy of a perfect $2 \times 3 \times 2$ supercell of BCC W with 24 atoms, $E_{\mathrm{He}}$ is the energy of an isolated $\mathrm{He}$ atom, and $E_{\mathrm{H}}$ is the total energy of an isolated $\mathrm{H}$ atom. The formation energy of various single point defects in $\mathrm{W}$ is summarized in Table 2, and the results are in good agreement with other calculation results $[1,20,21]$.

According to Table 2, we plotted the formation energies of the single point defect in Figure 4. For He, the substitutional site is the most favorable over the tetrahedral and octahedral sites. For $\mathrm{H}$, the tetrahedral site is the most preferred site over the substitutional and octahedral sites. Interestingly, the formation energies of $\mathrm{H}_{\mathrm{Tet}}$ and $\mathrm{H}_{\mathrm{Oct}}$ are negative, which implies that defects are more stable than other defects, and the formation process of the defects, $\mathrm{H}_{\mathrm{Tet}}$ and $\mathrm{H}_{\mathrm{Oct}}$, is an exothermic process. Figure 5 shows the formation energy of two $\mathrm{H}$ and $\mathrm{He}$ atoms in $\mathrm{W}$, and these two atoms are located in different sites. Figure 5(a) shows the formation energy of two $\mathrm{H}$ atoms that are located in different substitution, tetrahedral, and octahedral sites. According to the distance between two $\mathrm{H}$ atoms, they are marked as the first-, second-, and thirdnearest-neighbor atoms. Figure 5(b) shows the formation energy of two He atoms located in different substitutional, tetrahedral, and octahedral sites. For two $\mathrm{H}$ atoms, the highest formation energy is the $\mathrm{H}$ atoms located in different substitutional sites, and the lowest formation energy is closely related to the sites of the two $\mathrm{H}$ atoms. The formation energies of the two $\mathrm{H}$ atoms located in different octahedral sites are the lowest when the two $\mathrm{H}$ atoms are the first- or thirdnearest-neighbor atoms, the formation energy of the two $\mathrm{H}$ atoms that located in different tetrahedral sites is the lowest, and the two $\mathrm{H}$ atoms are the second-nearest-neighbor atoms. For He atoms, the lowest formation energy can be got when the two $\mathrm{He}$ atoms located in different substitutional sites. The formation energy of two He atoms located in different tetrahedral sites is the highest when the two He atoms are the first- or second-nearest-neighbor atoms. When two He atoms are the third-nearest-neighbor atoms, the highest formation energy can be got when the two He atoms located in different octahedral sites. The formation energy of the two foreign atoms $(\mathrm{H}$ or $\mathrm{He})$ is not equal to twice value of the single atom, and the formation energy of the two foreign atoms is two times smaller than the value of the formation energy of a 


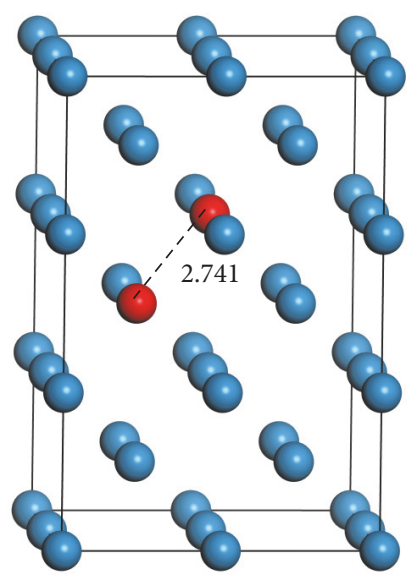

(a)

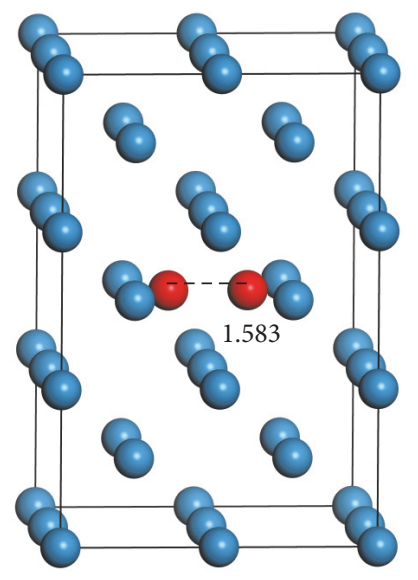

(d)

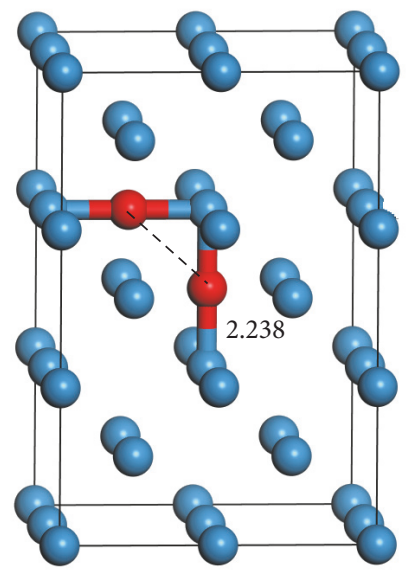

(g)

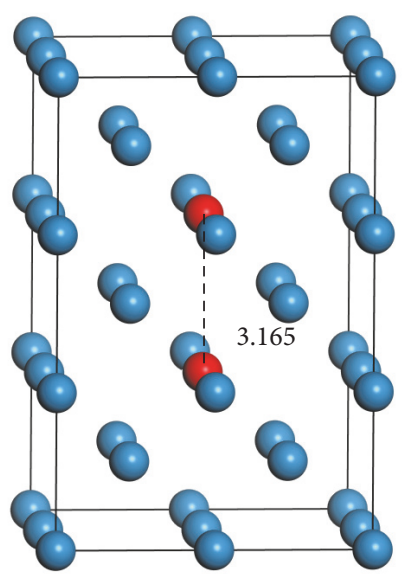

(b)

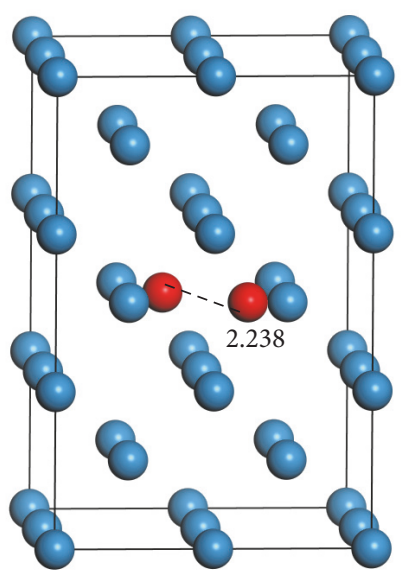

(e)

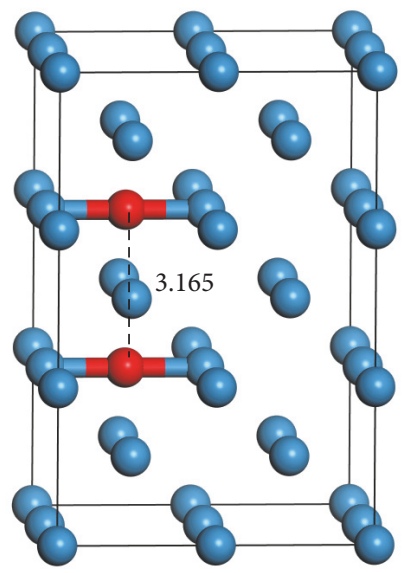

(h)

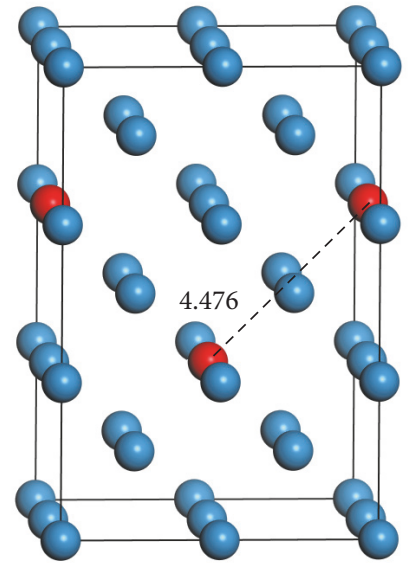

(c)

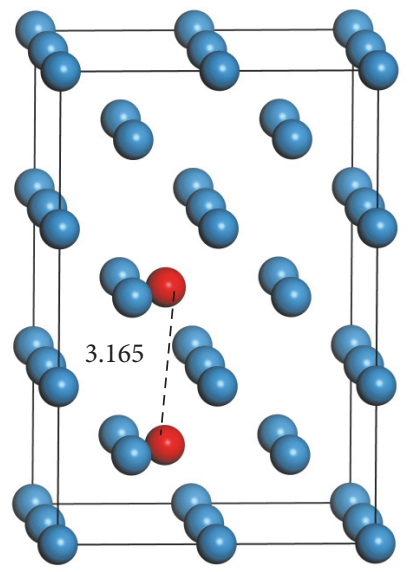

(f)

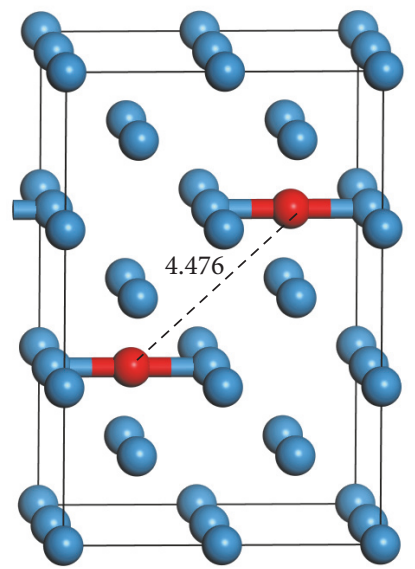

(i)

FIgure 2: The crystal structure of BCC W contains two $\mathrm{H}$ atoms or He atoms. The blue balls and red balls stand for W atoms and the $\mathrm{H}$ atoms or He atoms, respectively. (a), (b), and (c) show two atoms located in the substitutional site. (d), (e), and (f) show two atoms located in the tetrahedral site, and (g), (h), and (i) show two atoms located in the octahedral site. The numbers in the figure stand for the distances (in $\AA$ ) between the two foreign atoms. 
TABLE 1: Calculated and experimental lattice constants ( $a$ in $\AA$ ), elastic constants $\left(C_{i j}\right.$ in GPa), bulk modulus ( $B$ in GPa), Young's modulus ( $E$ in GPa), shear modulus ( $G$ in GPa), Poisson's ratio $(v)$, and $G / B$ for BCC W.

\begin{tabular}{lccccccccc}
\hline Parameters & $a$ & $C_{11}$ & $C_{12}$ & $C_{44}$ & $B$ & $E$ & $G$ & $v$ & $G / B$ \\
\hline This paper & 3.18 & 528.06 & 196.73 & 176.28 & 307.17 & 421.26 & 171.95 & 0.27 & 0.560 \\
Cal. [21] & 3.17 & 536.32 & 202.25 & 138.70 & 313.61 & 386.83 & 149.42 & 0.29 & 0.478 \\
Exp. [22] & 3.16 & 533 & 205 & 163 & 314.33 & 417.80 & 163.40 & 0.28 & 0.54 \\
\hline
\end{tabular}

TABLE 2: Summary of the formation energy of the single defect in W.

\begin{tabular}{lcccccc}
\hline Configuration & $\mathrm{H}_{\text {Sub }}$ & $\mathrm{H}_{\text {Tet }}$ & $\mathrm{H}_{\text {Oct }}$ & $\mathrm{He}_{\text {Sub }}$ & $\mathrm{He}_{\text {Tet }}$ & $\mathrm{He}_{\text {Oct }}$ \\
\hline This paper & 0.965 & -2.327 & -2.034 & 5.059 & 6.365 & 6.583 \\
Cal. [1] & 0.92 & -2.47 & -2.07 & 5.00 & 6.23 & 6.48 \\
Cal. [20, 21] & 0.78 & -2.44 & -2.06 & 4.70 & 6.16 \\
\hline
\end{tabular}

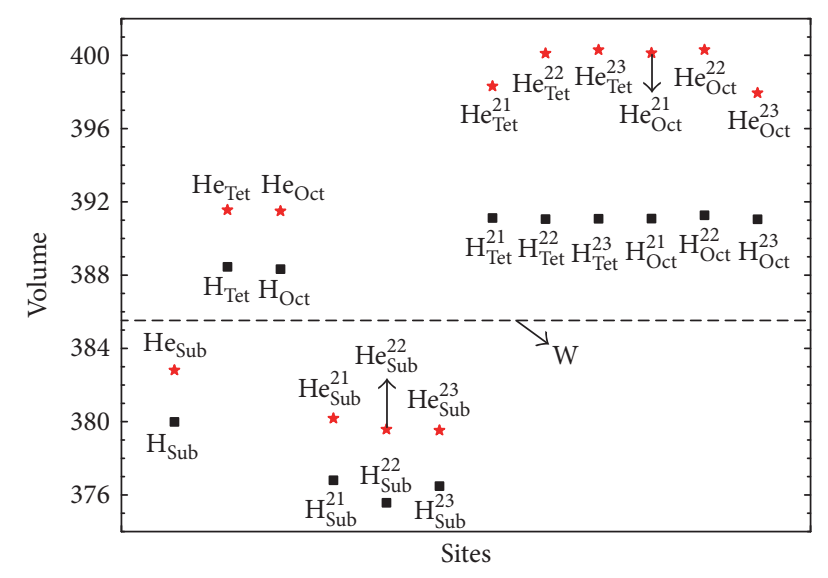

FIGURE 3: The volume (in $\left.\AA^{3}\right)$ of the supercell $(2 \times 3 \times 2)$ of the $\mathrm{W}$ supercell with single and two point defects formed by $\mathrm{H}$ and $\mathrm{He}$ atoms. The dashed line represents the volume of the perfect BCC W supercell.

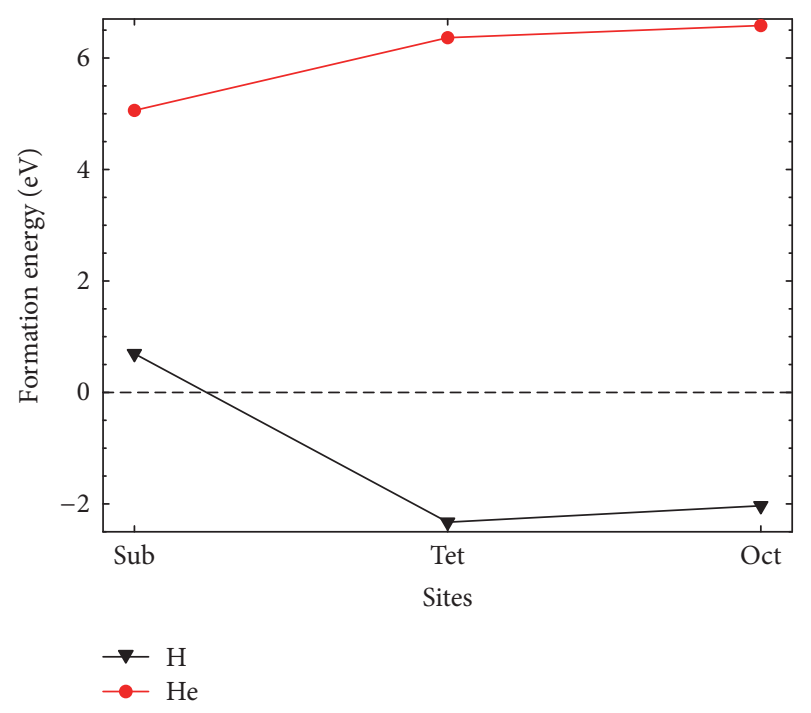

FIGURE 4: The formation energy of the single defects in W. single atom. In short, the formation energy of two $\mathrm{H}$ and $\mathrm{He}$ atoms are determined by their located sites.

3.3. Interaction of $\mathrm{H}-\mathrm{H}$ and $\mathrm{He}-\mathrm{He}$. The binding energy can reflect the interaction between two $\mathrm{H}$ or $\mathrm{He}$ atoms in W. The binding energy of two $\mathrm{H}$ or $\mathrm{He}$ atoms is calculated as follows:

$$
E_{\mathrm{b}}\left(X_{2}\right)=2 E(X)-E\left(X_{2}\right)-E_{\mathrm{W}}^{24}
$$

where $E(X)$ is the total energy of the $\mathrm{W}$ supercell with a $\mathrm{H}$ or He atom, $E\left(X_{2}\right)$ is the total energy of the supercell with two $\mathrm{H}$ or $\mathrm{He}$ atoms, and $E_{\mathrm{W}}^{24}$ is the total energy of $24 \mathrm{~W}$ atoms. A positive binding energy indicates an attraction exists between two atoms, while a negative binding energy shows a repulsion interaction exists between two atoms. The binding energies of two $\mathrm{H}$ or $\mathrm{He}$ atoms are shown in Figure 6. The most binding energies existing between two $\mathrm{H}$ atoms are near the zero while the most binding energies existing between two $\mathrm{He}$ atoms are in a positive value. The results show that there is an attraction interaction among the He atoms, and the interaction between two $\mathrm{H}$ atoms is very weak.

To further study the interaction between two $\mathrm{H}$ or $\mathrm{He}$ atoms, we recorded the distance between two $\mathrm{H}$ and $\mathrm{He}$ atoms before and after relaxation. Figure 7 shows the change of the distance between two $\mathrm{H}$ or $\mathrm{He}$ atoms after relaxation, and the atoms are located in different sites. The distance between two $\mathrm{H}$ atoms becomes longer after relaxation than the values before relaxation. This result suggests that a repulsive interaction exists between $\mathrm{H}$ atoms when the $\mathrm{H}$ atoms are close to each other, because each $\mathrm{H}$ atom in $\mathrm{W}$ lattice carries certain amount of negative charge. The distance between two $\mathrm{H}$ atoms is larger than $0.75 \AA$ (the distance between two $\mathrm{H}$ atoms in $\mathrm{H}_{2}$ molecule) after relaxation; these results indicate there is no $\mathrm{H}_{2}$ molecule formed. The result of our study is in good agreement with the results of Liu et al. [6]. The distance between two He atoms becomes shorter after relaxation. This result indicates that there is an attraction interaction between the two $\mathrm{He}$ atoms when they are close to each other. 


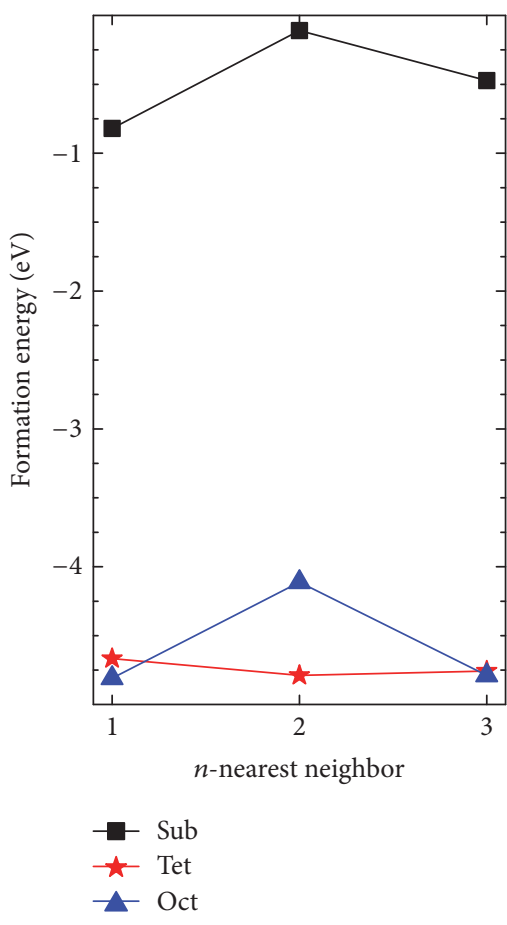

(a)

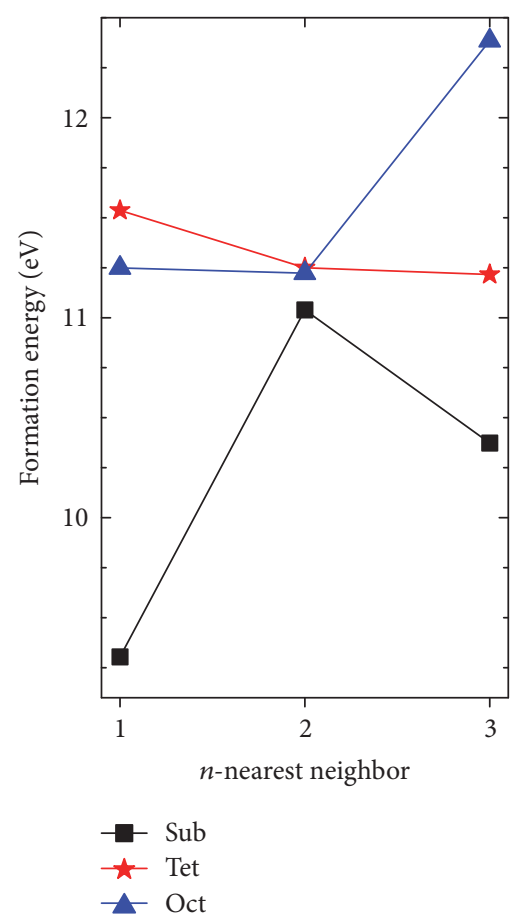

(b)

Figure 5: The formation energy of two $\mathrm{H}(\mathrm{a})$ and $\mathrm{He}(\mathrm{b})$ atoms located in the different sites.

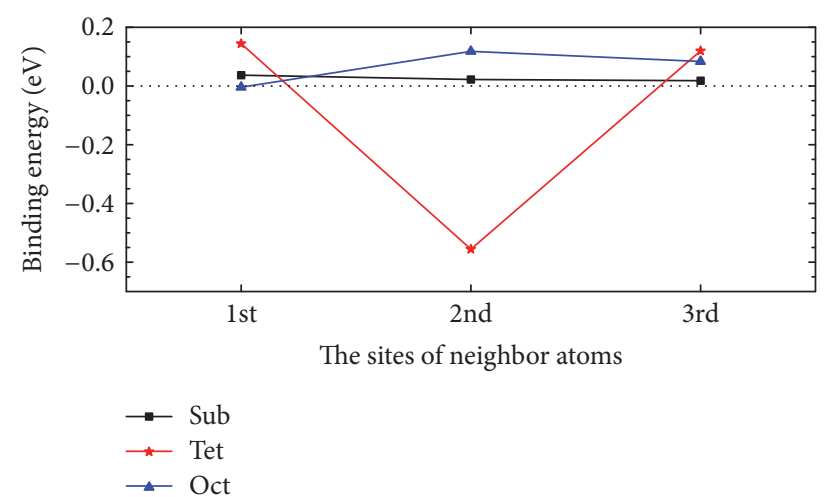

(a)

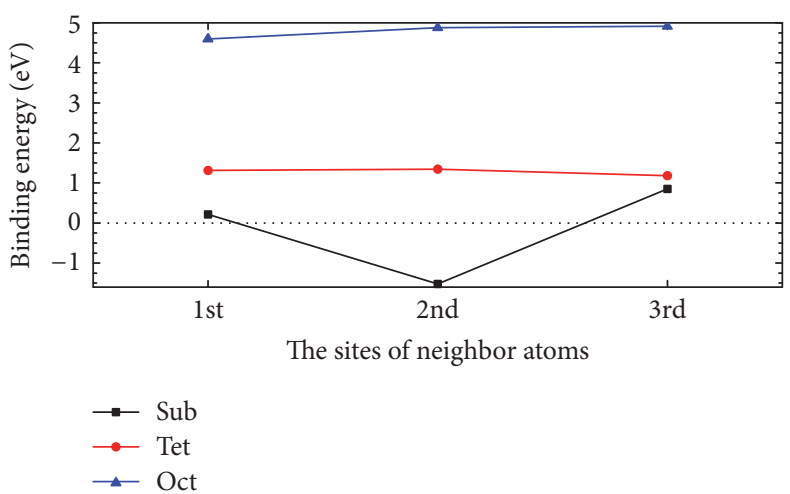

(b)

Figure 6: The binding energy of two $\mathrm{H}$ (a) and $\mathrm{He}(\mathrm{b})$ atoms in $\mathrm{W}$ when they are located in different positions and the distance of two $\mathrm{H}$ and He atoms is increased.

\subsection{Analysis of the Mulliken Population and Charge Density.} The electronic structure can help us understand the root causes of the interaction between two $\mathrm{H}$ and $\mathrm{He}$ atoms, so the Mulliken population and the charge density were analyzed in this paper. Table 3 shows the atomic population of the $\mathrm{H}$ and $\mathrm{He}$ atoms in $\mathrm{W}$, and two $\mathrm{H}$ and $\mathrm{He}$ atoms are the first-nearest-neighbor atoms. Mulliken population analysis shows that certain electrons will transfer from $\mathrm{W}$ to $\mathrm{H}$, and $\mathrm{H}$ atoms can stand in a stable state. The $\mathrm{H}$ atoms will accept 0.10 electrons from the surrounding $\mathrm{W}$ atoms when two $\mathrm{H}$ atoms are located in substitutional sites. The $\mathrm{H}$ atoms accept 0.24 electrons when two $\mathrm{H}$ atoms are located in the tetrahedral sites, and they accept 0.26 electrons when they are located in the octahedral sites. The amount of charge transfer is insensitive to the interstitial $\mathrm{H}$ and $\mathrm{He}$ atoms' positions. The $\mathrm{H}$ atoms have a negative charge. This implies that there is a Coulomb repulsion between two $\mathrm{H}$ atoms. On the contrary, $\mathrm{W}$ will get some electrons from the He atoms. $\mathrm{W}$ atoms will accept 0.05 electrons from a $\mathrm{He}$ atom when the $\mathrm{He}$ atoms are located in the substitution sites; $\mathrm{W}$ atoms will get 0.11 electrons from a He atom when $\mathrm{He}$ atoms are located in the tetrahedral and octahedral sites. Thus, the Van der Waals force between two He atoms becomes stronger when they are close to each other. The more electrons the $\mathrm{W}$ atoms get, the more stable the structure is. Interestingly, we found that the $\mathrm{H}-\mathrm{H}$ repulsive interaction fits well to the screened 


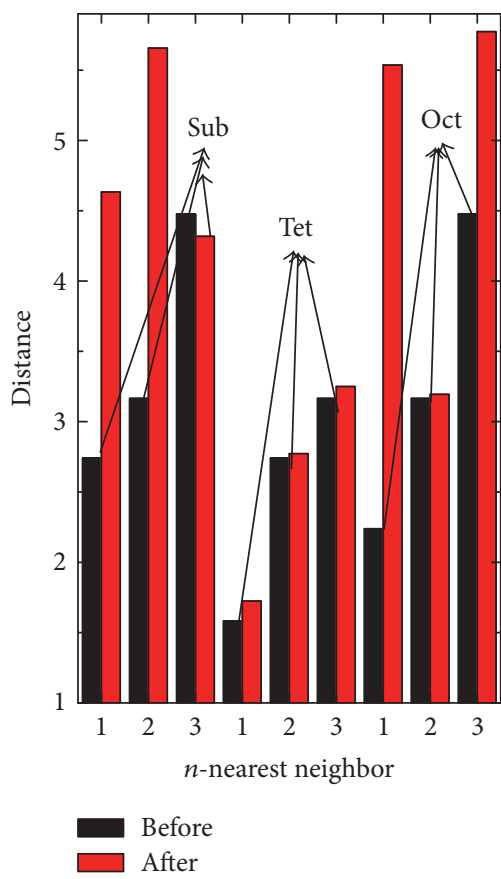

(a)

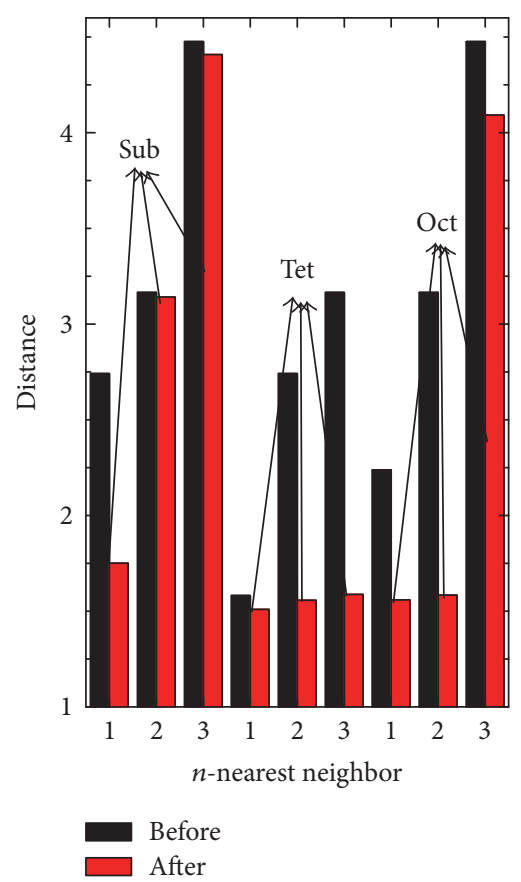

(b)

FIGURE 7: The distance (in $\AA$ ) between the two H (a) or He (b) atoms before the relaxation and after relaxation.

TABLE 3: Atomic populations (Mulliken) of $\mathrm{H}$ and $\mathrm{He}$ atoms in $\mathrm{W}$.

\begin{tabular}{lcccc}
\hline Sites & Species & Ion & $s$ & Charge $(e)$ \\
\hline $\mathrm{H}_{\text {Sub }}^{21}$ & $\mathrm{H}$ & 1 & 1.10 & -0.10 \\
& $\mathrm{H}$ & 2 & 1.10 & -0.10 \\
$\mathrm{He}_{\text {Sub }}^{21}$ & $\mathrm{He}$ & 1 & 1.95 & 0.05 \\
& $\mathrm{He}$ & 2 & 1.95 & 0.05 \\
$\mathrm{H}_{\text {Tet }}^{21}$ & $\mathrm{H}$ & 1 & 1.24 & -0.24 \\
& $\mathrm{H}$ & 2 & 1.10 & -0.24 \\
$\mathrm{He}_{\text {Tet }}^{21}$ & $\mathrm{He}$ & 1 & 1.89 & 0.11 \\
& $\mathrm{He}$ & 2 & 1.89 & 0.11 \\
$\mathrm{H}_{\text {Oct }}^{21}$ & $\mathrm{H}$ & 1 & 1.26 & -0.26 \\
& $\mathrm{H}$ & 2 & 1.26 & -0.26 \\
$\mathrm{He}_{\text {Oct }}^{21}$ & $\mathrm{He}$ & 1 & 1.89 & 0.11 \\
& $\mathrm{He}$ & 2 & 1.89 & 0.11 \\
\hline
\end{tabular}

Coulomb potential. To explain the differences between the interactions between two $\mathrm{H}$ atoms and $\mathrm{He}$ atoms, charge density distribution was used. We selected a single atom and two atoms located in the octahedral site. Figure 8 shows the charge density of one and two atoms. Figures 8(a) and 8(d) show the charge density of single $\mathrm{H}$ and $\mathrm{He}$ atom in $\mathrm{W}$, respectively. Figures $8(\mathrm{~b})$ and $8(\mathrm{c})$ show two $\mathrm{H}$ atoms in $\mathrm{W}$, and Figures 8(e) and 8(f) also show two He atoms in W from several different perspectives. The bonds between $\mathrm{H}$ and $\mathrm{W}$ contain covalent components. The bond is stronger than that between $\mathrm{He}$ and $\mathrm{W}$, and the charge density difference between $\mathrm{H}$ and $\mathrm{W}$ is bigger than that between $\mathrm{He}$ and $\mathrm{W}$. The $\mathrm{H}$ atoms are more likely to be attracted by $\mathrm{W}$ atoms than by each other while the He atoms are likely to attract each other. There is only one electron in the $1 s$ orbital of an $\mathrm{H}$ atom, and the $\mathrm{H}$ atom obtains the electrons from the $\mathrm{W}$ atoms to maintain a relatively stable state. He atom is a closed shell atom. It prefers to occupy the low electron density zone (interstitial sites).

\section{Conclusion}

Using first-principles calculation, we studied the BCC W with single and two point defects formed by $\mathrm{H}$ or $\mathrm{He}$ atoms, respectively. The results of our calculations are as follows:

(1) For a single $\mathrm{H}$ atom, the most preferred site is the tetrahedral interstitial site. For He, the substitutional site is more favorable than the tetrahedral and octahedral sites.

(2) For two $\mathrm{H}$ and $\mathrm{He}$ atoms, the formation energy is closely related to the sites of the two atoms. The formation energy of two $\mathrm{H}$ atoms that were located in different substitutional sites is the highest, while the formation energy of $\mathrm{He}$ atoms is lowest when the two $\mathrm{He}$ atoms are located in different substitutional sites.

(3) After relaxation, two $\mathrm{H}$ atoms are away from each other because the $\mathrm{H}$ atom accepts some charge from the surrounding $\mathrm{W}$ atoms and they form a covalent bond. The He atoms are close to each other because the $\mathrm{W}$ atoms get some electrons from the He atoms, and the more electrons the $\mathrm{W}$ atoms get, the more stable the crystal structure is.

Our results are good in agreement with other simulation results and experimental data. This paper might shed some 


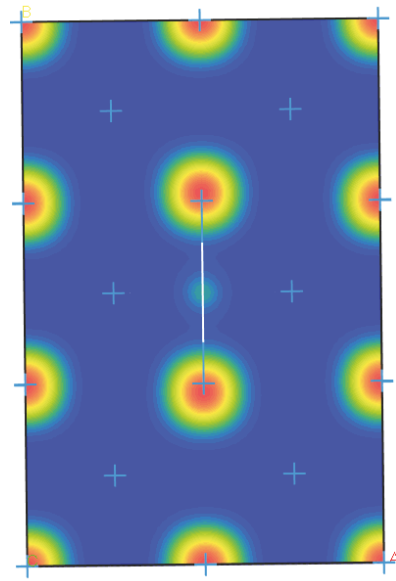

(a)

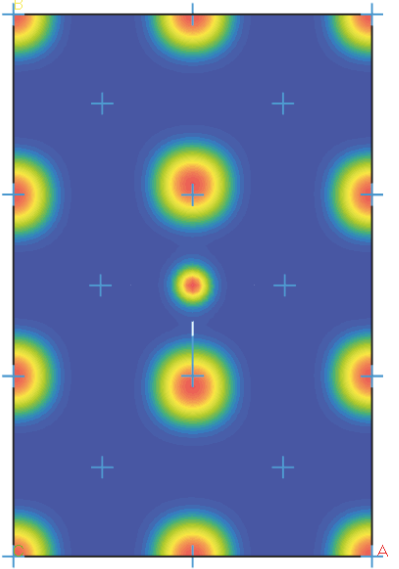

(d)

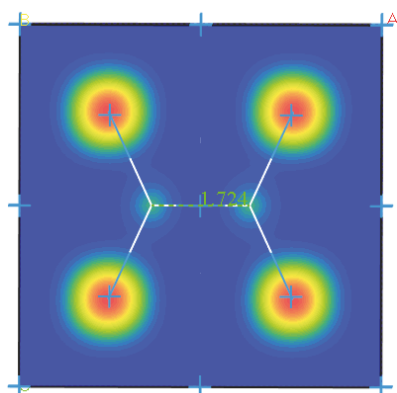

(b)

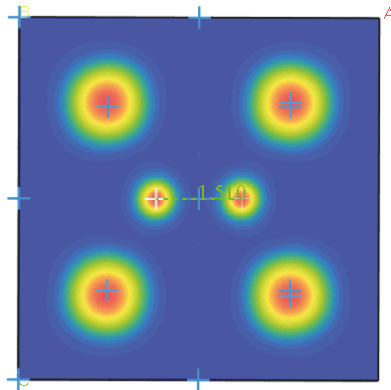

(e)

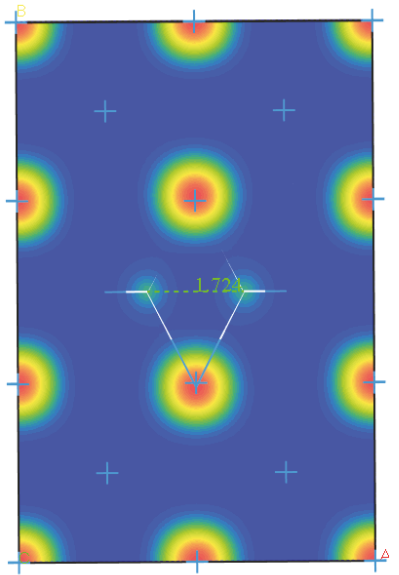

(c)

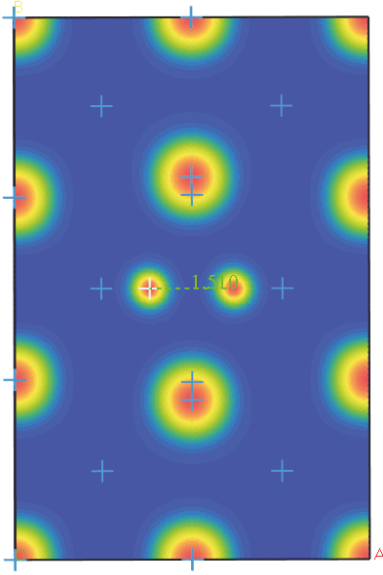

(f)

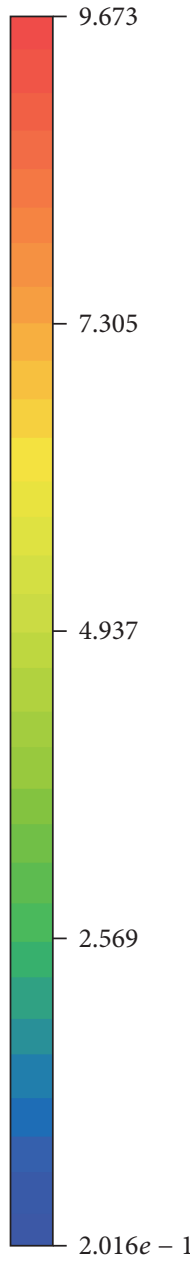

$2.016 e-1$

Figure 8: Charge density distribution. (a), (b), and (c) show the single $\mathrm{H}$ atom and two $\mathrm{H}$ atoms located in octahedral sites; (d), (e), and (f) show the single He atom and two He atoms located in different octahedral sites. The red color represents high charge density, and the blue color represents low valence charge density.

light on the development of tungsten materials used in ITER and other fusion reactors.

\section{Conflicts of Interest}

The authors declare that there are no conflicts of interest regarding the publication of this paper.

\section{Acknowledgments}

This work was supported by the Fundamental Research Funds for the Central Universities under Grant no. 2017 MS079.

\section{References}

[1] S. C. Lee, J. H. Choi, and J. G. Lee, "Energetics of He and H atoms with vacancies in tungsten: First-principles approach," Journal of Nuclear Materials, vol. 383, no. 3, pp. 244-246, 2009.
[2] C. H. Skinner, A. A. Haasz, V. K. H. Almov et al., "Recent advances on hydrogen retention in iter's plasma-facing materials: Beryllium, carbon. And tungsten," Fusion Science and Technology, vol. 54, no. 4, pp. 891-945, 2008.

[3] I. I. Arkhipov, S. L. Kanashenko, V. M. Sharapov, R. K. Zalavutdinov, and A. E. Gorodetsky, "Deuterium trapping in iondamaged tungsten single crystal," Journal of Nuclear Materials, vol. 363-365, no. 1-3, pp. 1168-1172, 2007.

[4] Y. M. You, "Effects of low energy and high flux helium/hydrogen plasma irradiation on tungsten as plasma facing material," Plasma Science and Technology, vol. 7, no. 3, pp. 2828-2834, 2005.

[5] P. Grigorev, D. Terentyev, V. Dubinko et al., "Nucleation and growth of hydrogen bubbles on dislocations in tungsten under high flux low energy plasma exposure," Nuclear Instruments and Methods in Physics Research, Section B: Beam Interactions with Materials and Atoms, vol. 352, pp. 96-99, 2015.

[6] Y. L. Liu, W. Lu, A. Y. Gao, L. J. Gui, and Y. Zhang, "Firstprinciples investigation of diffusion behaviours of $\mathrm{H}$ isotopes: 
from W(110) surface into bulk and in bulk W,' Chinese Physics $B$, vol. 21, no. 12, 2012.

[7] X. X. Wang, Y. Zhang, H. B. Zhou, and J. L. Wang, "Effects of niobium on helium behaviors in tungsten: a first-principles investigation," Acta Physica Sinica, vol. 63, no. 4, article 046103, 2014.

[8] M. Li, J. Cui, J. Wang, and Q. Hou, "Molecular dynamics simulations of cumulative helium bombardments on tungsten surfaces," Nuclear Instruments and Methods in Physics Research, Section B: Beam Interactions with Materials and Atoms, vol. 337, pp. 45-54, 2014.

[9] Q. Zhao, Z. Zhang, Y. Li, and X. P. Ouyang, "The mechanical and thermodynamic properties of $\beta$-si ${ }_{1-x} c_{x}$," RSC Advances, vol. 7, no. 45, pp. 28499-28505, 2017.

[10] D. Y. Jiang, Z. Zhang, R. X. Liang et al., "Electronic structure and optical properties of the scintillation material wurtzite ZnS(Ag)," Nuclear Science and Techniques, vol. 28, no. 3, pp. 3541, 2017.

[11] Z. Zhang, Q. Zhao, Y. Li, and X. P. Ouyang, "Electronic structure and optical properties of CsI, CsI(Ag), and CsI(Tl)," Journal of the Korean Physical Society, vol. 68, no. 9, pp. 1069-1074, 2016.

[12] J. Xu and J. Zhao, "First-principles study of hydrogen in perfect tungsten crystal," Nuclear Instruments and Methods in Physics Research, Section B, vol. 267, no. 18, pp. 3170-3174, 2009.

[13] L. Cheng, G. De Temmerman, T. W. Morgan et al., "Mitigated blistering and deuterium retention in tungsten exposed to highflux deuterium-neon mixed plasmas," Nuclear Fusion, vol. 57, no. 4, article 046028, 2017.

[14] D. Nguyen-Manh and S. L. Dudarev, “Trapping of He clusters by inert-gas impurities in tungsten: First-principles predictions and experimental validation," Nuclear Instruments and Methods in Physics Research, Section B, vol. 352, pp. 86-91, 2015.

[15] E. V. Kornelsen and A. A. Van Gorkum, "Study of bubble nucleation in tungsten using thermal desorption spectrometry: clusters of 2 to 100 helium atoms," Journal of Nuclear Materials, vol. 92, no. 1, pp. 79-88, 1980.

[16] P. Hohenberg and W. Kohn, "Inhomogeneous electron gas," Physical Review, vol. 136, no. 3B, pp. B864-B871, 1964.

[17] S. Clark, M. Segall, C. Pickard, P. Hasnip, M. Probert, and K. Refson, "First principles methods using castep," Zeitschrift für Kristallographie, vol. 13, pp. B864-B871, 1964.

[18] J. P. Perdew and W. Yue, "Accurate and simple density functional for the electronic exchange energy: generalized gradient approximation," Physical Review B, vol. 33, no. 12, pp. 88008802, 1986.

[19] J. P. Perdew, K. Burke, and M. Ernzerhof, "Generalized gradient approximation made simple," Physical Review Letters, vol. 77, no. 18, pp. 3865-3868, 1996.

[20] Y. L. Liu, Y. Zhang, G. N. Luo, and G. H. Lu, "Structure, stability and diffusion of hydrogen in tungsten: a first-principles study," Journal of Nuclear Materials, vol. 390-391, no. 1, pp. 1032-1034, 2009.

[21] C. S. Becquart and C. Domain, "Ab initio calculations about intrinsic point defects and He in W," Nuclear Instruments and Methods in Physics Research, Section B, vol. 255, no. 1, pp. 2326, 2007.

[22] P. Söderlind, O. Eriksson, J. M. Wills, and A. M. Boring, “Theory of elastic constants of cubic transition metals and alloys," Physical Review B, vol. 48, no. 9, pp. 5844-5851, 1993. 


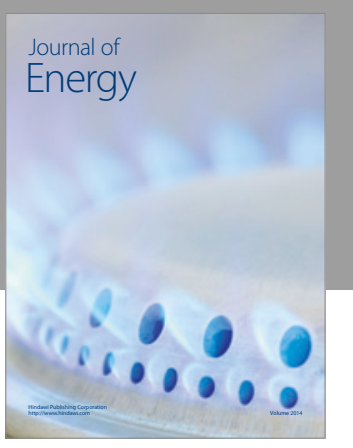

Journal of

Industrial Engineering
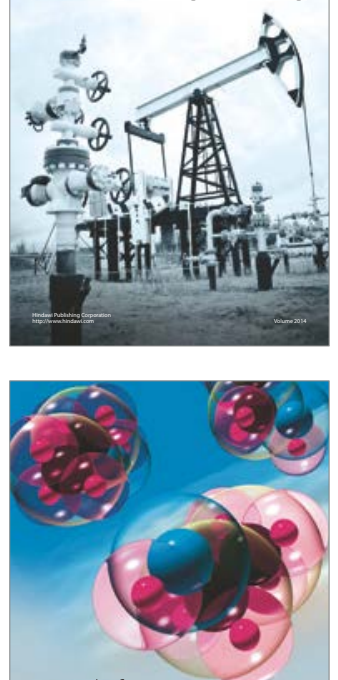

Fuels
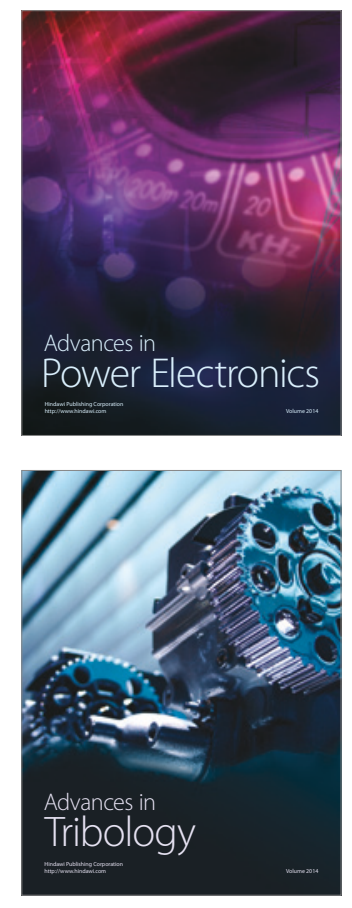
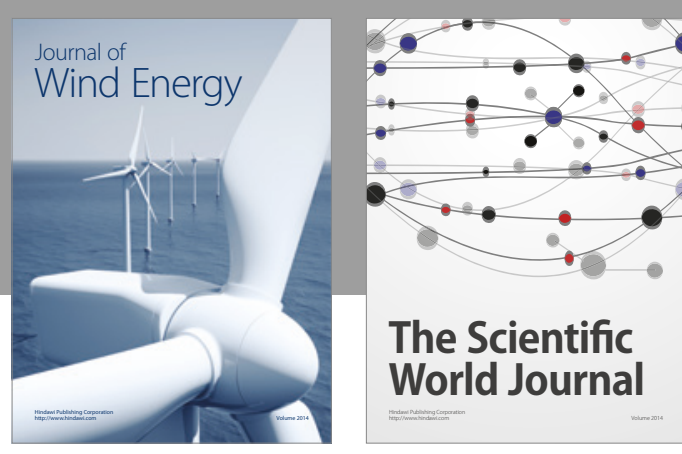

The Scientific World Journal
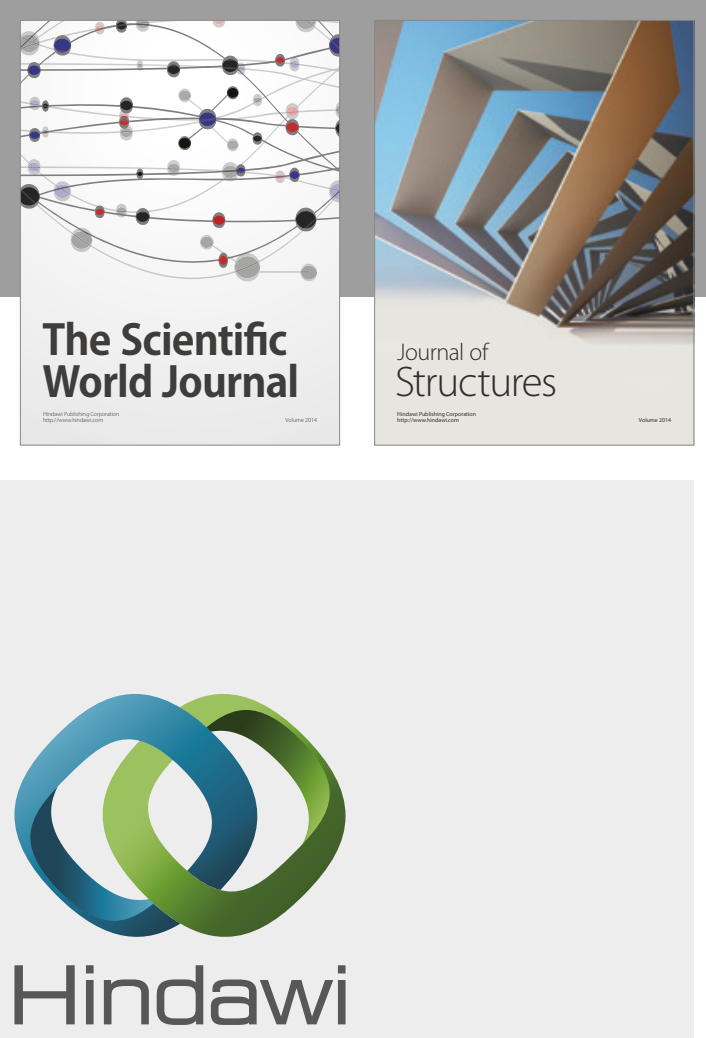

Submit your manuscripts at

https://www.hindawi.com
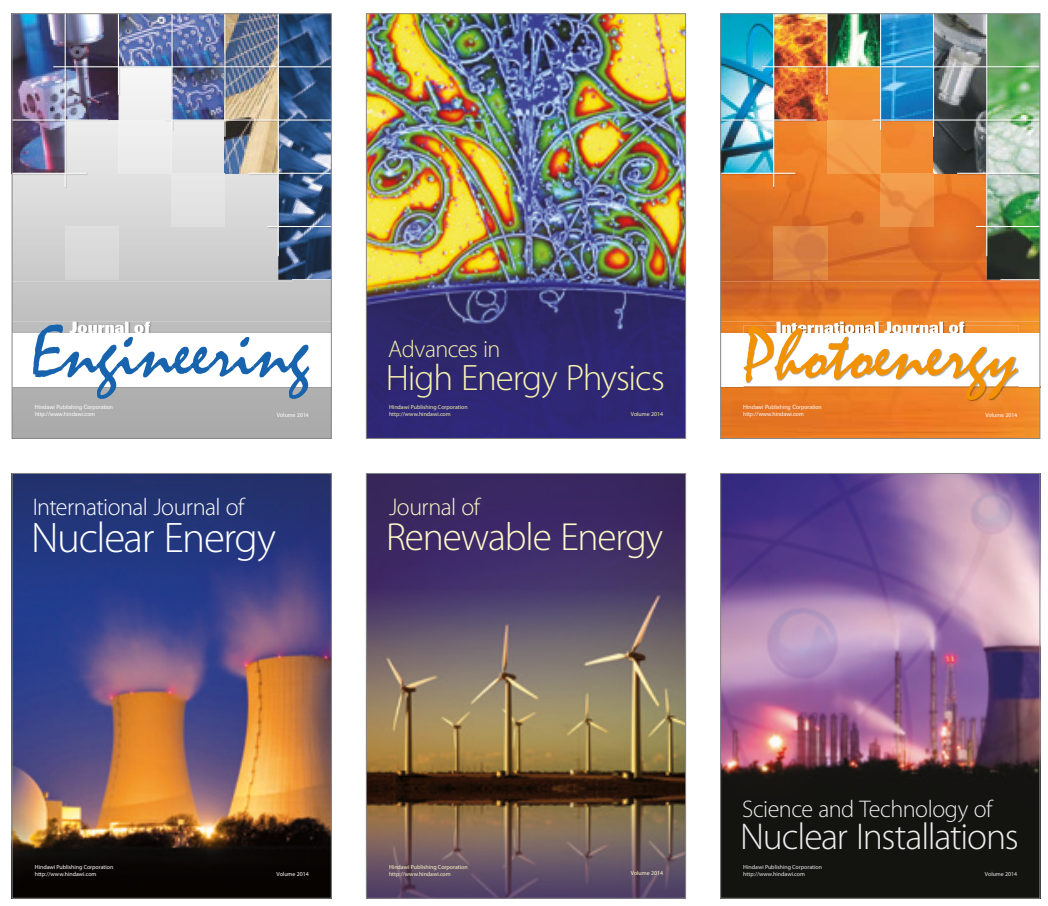

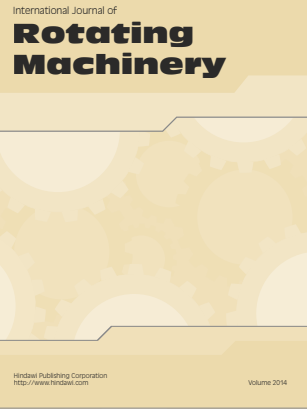

Journal of

Petroleum Engineering

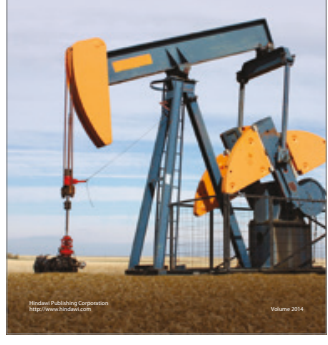

Journal of
Solar Energy
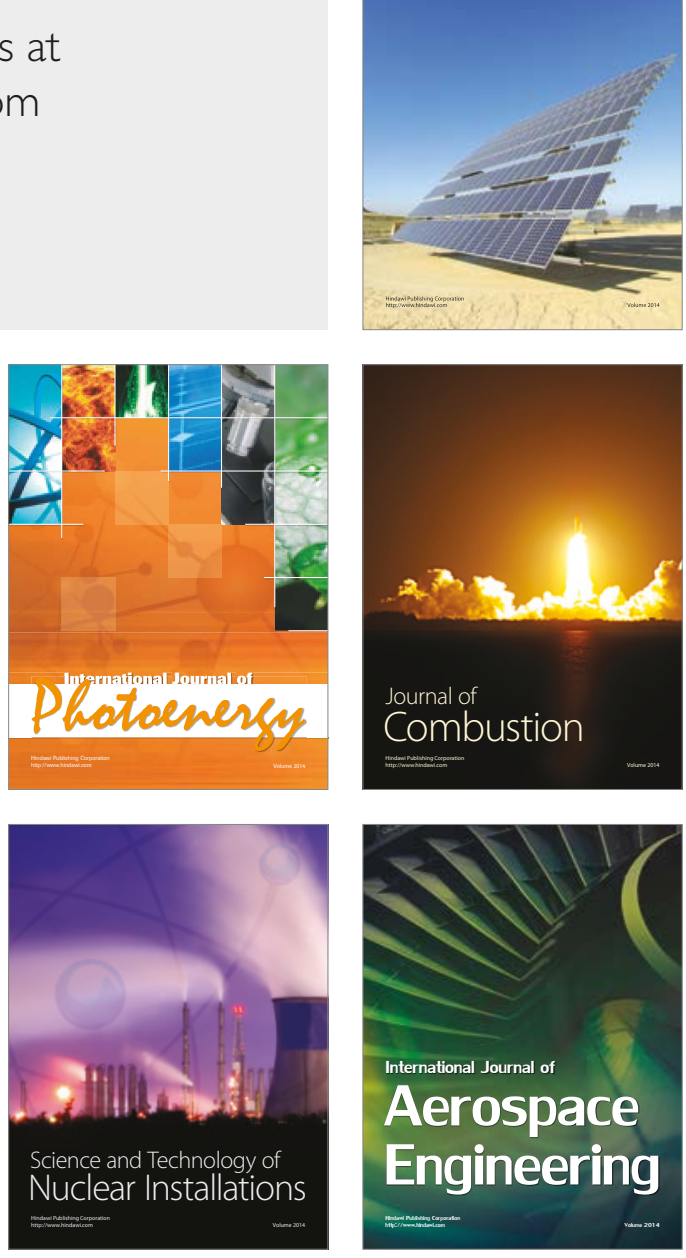